\title{
Dealing with Resource Requirement in Multimedia Document Consistency Verification
}

\author{
A.Abdelli \\ Computer Science Department- Bp 32 El-Alia babezzouar Algiers Algeria 16111- \\ Tel \Fax: +21321247917 \\ Email:Abdelli@lsi-usthb.dz
}

\begin{abstract}
The paper proposes to revisit the concept of multimedia document consistency. Within this context, numerous works have addressed approaches for the temporal consistency verification of such documents, especially those that are encoded using the SMIL language, but to our best knowledge, no one has proposed to handle the resource conflict issue and its impact on both multimedia presentation quality and consistency. Therefore, we propose to work out this issue by taking into account the resource needs when carrying out the consistency verification. Hence, a new paradigm called "qualitative consistency" is defined improving the previous concepts already defined for this topic.
\end{abstract}

Keywords: Multimedia document, Time Petri Nets, Consistency verification.

\section{INTRODUCTION}

The formal verification of the temporal consistency of multimedia documents has been widely addressed in the literature through different approaches [2][10][7][9][14]. Actually, the mainstream techniques [14][13] are those based on reachability analysis by using formal models that allow capturing the time requirements as well as the synchronisation constraints of the multimedia presentation. Then, a reachability graph is derived thereof encompassing all possible temporal scenarios of the presentation. The analysis of these scenarios is carried out by scrolling up all the paths in the graph to detect potential inconsistencies. Hence, the localisation of the origin of the errors makes it possible at the authoring stage to correct them by temporal formatting of the document code. In addition, the graph could be exploited at the player stage as a decision tool to schedule an appropriate scenario among consistent ones.

However, to the best of our knowledge, there has been no report in the literature that handles resource conflict issue when dealing with the consistency verification of multimedia document. To be explicit, let us consider the SMIL language [15] which has become the most dominant reference on the internet for characterizing complex distributed multimedia presentations. These documents that allow to organize the spacio-temporal fitting of several media presentations, need specific resources (e.g screen or sound device) to let the driven information be user-perceived. In resource conflict situations, a priority policy is set by the player and operates in real time to determine which element will obtain the required resource. Consequently, the resource acquisition may provoke the preemption of lesser priority presentations. Hence, a part of, even the whole presentation of at least one media will not be delivered to the user leading to a loss of information which influences badly the quality and the consistency of the multimedia presentation.

To handle this specific issue, we have proposed in a recent work [3] a time Petri net based model that allows describing accurately the resource allocation schemes that are observed in multimedia presentation. Then, we carried on our proposal by addressing in [4] a thorough modeling process making it possible to handle multimedia requirements when assuming a Real one player like functioning [12]. Hence, with the support of the approach developed in [5] we are able from 
this TPN specification [4] to derive a reachability graph that encompasses all possible behaviors highlighting the conflict situations by means of three special events (strong event, violated event, and the violator event).

We propose in this paper to carry on our verification methodology by revisiting the consistency verification concept introduced in [14][13]. Our proposal aims to take into account the resource conflict issue when performing the consistency verification. We achieve this goal by analyzing all possible runs in terms of event sequences extracted from the reachability graph; With the support of an appropiate example, we show how to detect inconsistencies owed to resource conflicts when they occur, and how we can cure them. Hence, a new verification methodology called "Qualitative consistency verification" is formally introduced.

The remainder of this paper is organized as follows. Section 2 introduces briefly the temporal consistency verification methodology through an example. In Section 3, we describe clearly the issue related to resource conflicts while emphasizing its impact on multimedia document consistency. Finally, Section. 4 introduces the "qualitative consistency" methodology verification.

\section{TEMPORAL CONSISTENCY VERIFICATION OF MULTIMEDIA DOCUMENTS}

A multimedia document can be seen as the fitting of different basic media presentations (video, audio, text, image or animation) that progress according to a certain scheme (in parallel or in sequential) while being synchronized, time constrained, and may being conflicted for the same resources. Therefore, theses complex schemes may yield inconsistent presentation when processing the document by the player. However, the consistency verification of the document ${ }^{1}$ can help to avoid these misadventures by using formal methodologies. To be more explicit, the formal verification of a multimedia document is performed by capturing its constraints thanks to the use of a formal model. Then, a reachability graph is derived from the former making it possible to depict all possible scenarios of the multimedia presentation; highlighting potential errors when and where they occur ${ }^{2}$. Hence, it becomes possible within an authoring tool to correct these incoherences by formatting the original document, and within the player tool to schedule only consistent scenarios when they exist.

To illustrate this verification technique, let us consider the SMIL encoded multimedia document depicted in Fig. 1. This presentation consists on a sequence $s e q_{1}$ of an image "Img $g_{1}$ " followed by the parallel presentation of an audio clip "Aud ${ }_{1}$ " and a text "Txt". This sequence must be presented simultaneously with two other sequences: The first of the two sequences $s e q_{2}$ shows a video clip "Vid ${ }_{1}$ " followed by the parallel presentation "par ${ }_{2}$ " of an image "Img ${ }_{2}$ " and a double repetition of an audio clip "Aud ${ }_{2}$ ". The second sequence "seq ${ }_{3}$ " presents a video "Vid 2 " that begins " $4 s^{\prime}$ "after

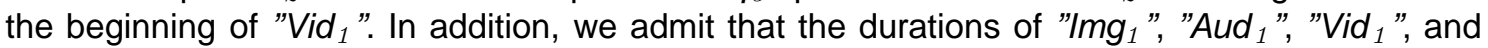
"Aud ${ }_{2}$ " are, respectively, 5, 10, 8 and 5 seconds, and that the medias "Img ${ }_{2}$ ", "Txt" and "Vid 2 " do not have explicit durations. It is assumed besides that the start of the bloc "par 2 " conditions the end of the media "Aud ${ }_{1}$ " and that "Aud 2 " must begin once the media "Aud 1 " ends. Furthermore, the media " $\mathrm{Vid}_{1}$ " can be ended by a user interaction, if it occurs between 4 and 6 seconds after the beginning of "Vid ${ }_{1}$. . Also, noteworthy that the end of the sequence "seq ${ }_{2}$ " is conditioned by the end of "Aud 2 " and the end of the SMIL presentation is determined by the end of the sequence $\mathrm{seq}_{2}$. Hence, the SMIL presentation finishes when the ending event related to "Aud ${ }_{2}$ " occurs.

The formal verification of the consistency of a multimedia document has been treated in [13]. There, a document is said to be temporally consistent: "if the action characterizing the beginning of the presentation is necessarily followed (and this for every path considered in the graph) by an action characterizing its end".

For instance, the Fig.2 shows the reachability graph of the previous $S M I L$ presentation, obtained when using our approach which is based on Time Petri nets specification [4]. Each edge of the

\footnotetext{
${ }^{1}$ Generally, the verification is performed earlier at the authoring stage, or at the player stage.

${ }^{2}$ In almost all the errors are due to a bad characterization of the document at the authoring stage
} 


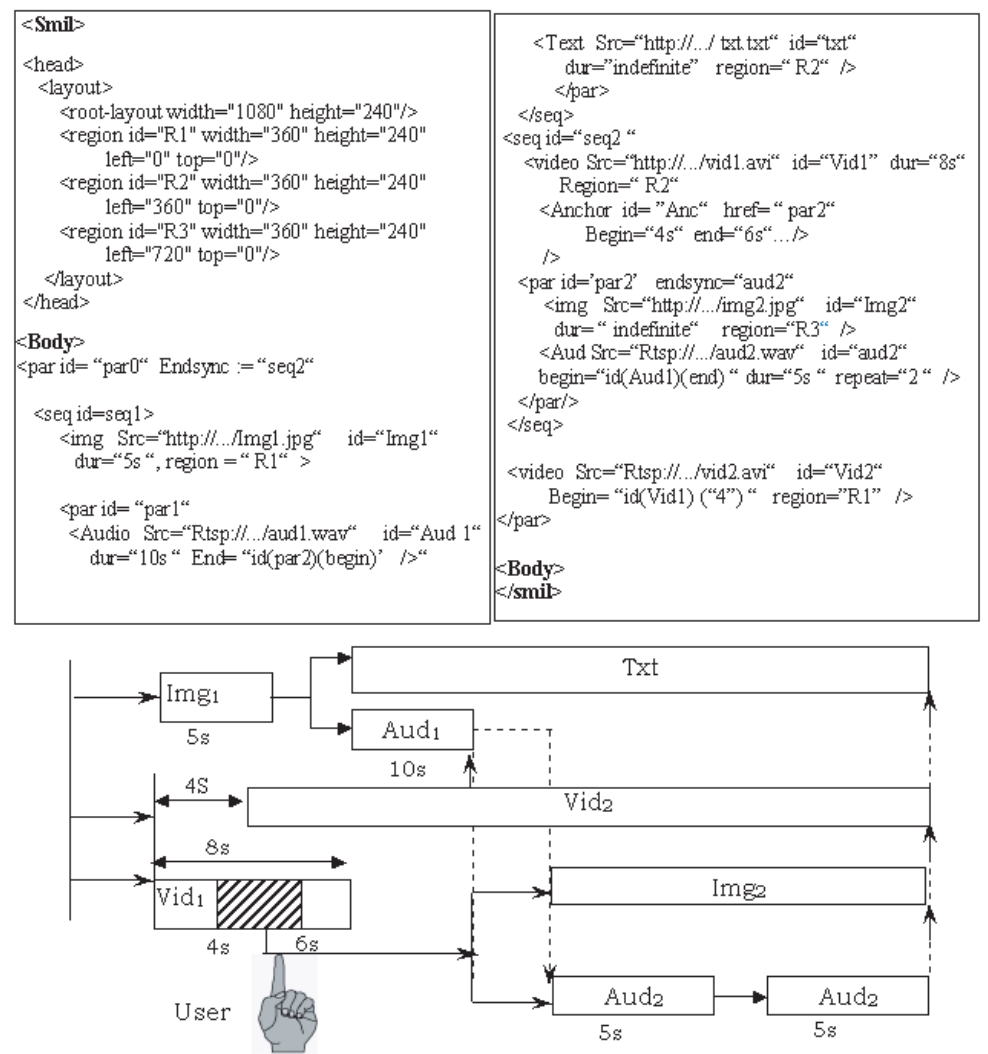

FIGURE 1: A multimedia presentation and its corresponding SMIL code.

graph is labeled with the set of parallel events occurring for the same dates, whereas the nodes contain marking and time information. The events can denote:

- the begin or the end of a media noted respectively $B(O)$ and $E(O)$,

- the occurrence of a user interaction Anc,

- actions that can help to design synchronization for instance $\left\{\operatorname{Syn}\left(\mathrm{Aud}_{1}, \mathrm{Aud} \mathrm{d}_{2}\right), \operatorname{Syn}\left(\operatorname{Vid}_{1}, \operatorname{Vid}_{2}\right), B\left(\mathrm{Par}_{2}\right)\right\}$,

- or actions that specify specific events like the occurrence of a repetition of a same media $\left\{\operatorname{Occ}\left(\mathrm{Aud}_{2}\right)\right\}$.

As regards temporal consistency of this document, one can notice that paths $0 \longrightarrow 6,0 \longrightarrow 11$ and $0 \longrightarrow 15$ are not consistent since the set of events ending these paths do not involve any occurrence of the event $E\left(A_{u d} d_{2}\right)$. These paths describe inconsistent scenarios resulted when the user interaction intervenes between 4 and before 5 seconds, after beginning " $V$ id $_{1}$ ". In these situations, the bloc "par " starts before the object "Aud ${ }_{1}$ " which keeps the latter unable to achieve its own presentation ${ }^{3}$. Therefore, the media "Aud 2 " is not allowed to start, since "Aud ${ }_{1}$ " has not finished its own presentation ${ }^{4}$. The remaining paths leading to node 20 describe consistent scenarios of the multimedia presentation. For instance, the path $0 \longrightarrow 13 \longrightarrow 20$ depicts the case where the user interaction intervenes at date 5 s, whereas the path $0 \longrightarrow 22 \longrightarrow 20$ models the run within which the user acts after $5 s$ and before $6 s$. The last scenario is designed by the path $0 \longrightarrow 26 \longrightarrow 20$, and denotes the case where the user does not interact with the presentation yielding the occurrence of the timeout event Tout $(A n c)^{5}$.

\footnotetext{
${ }^{3}$ With respect of the synchronisation value of the ending event of $A u d_{1}$

${ }^{4}$ According to the synchronization linked to the start of "Aud 2

${ }^{5}$ The occurrence of this event denotes that the link is no longer displayed and therefore the user wil not be able to interact with the presentation afterwards.
} 


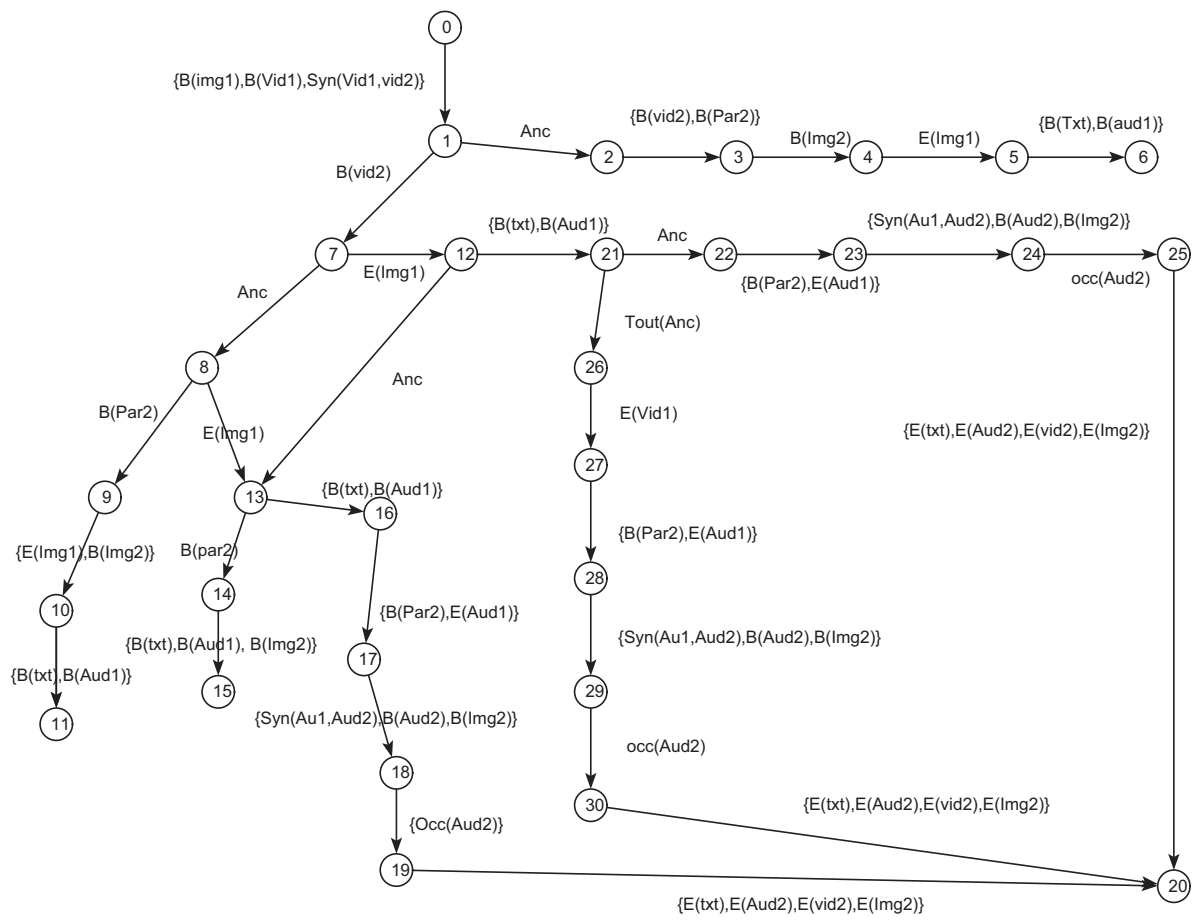

FIGURE 2: The reachability graph depicting the time and the synchronization requirements of the SMIL multimedia presentation.

Although the previous document is temporally inconsistent, its reachability graph can be used at player level as a decision tool to schedule only consistent scenarios. Therefore, it needs to remove all the inconsistent paths in the graph, which in our case entails to force the user interaction to occur at least 5 seconds after the start of the media "Vid ${ }_{1}$ ". Thus, the player can inhibit the user interaction between 4 and 5 seconds while it allows its occurrence after that.

However, the previous methodology permits only to check for temporal inconsistencies since at the origin the modeling process deals only with time and synchronization requirements of the document. Other concerns like resource conflict may have also an impact on the consistency verification, but need however additional mechanisms in order to be handled during the modeling process. Moreover, for this effect the consistency validation approach should also be revisited while including these concerns. Consequently, to achieve this goal, we discuss thereafter this issue and propose a new consistency verification technique to work it out.

\section{DEALING WITH RESOURCE CONFLICT}

As we noticed previously, a multimedia presentation can deal with resource conflicts which may induce dysfunctions when processing it. The later issue is still considered as a secondary concern, and has not been handled seriously in the literature yet. Therefore, we investigate thereafter to study the impact of this issue on multimedia presentation consistency.

For that effect, note recalling that multimedia presentations and especially $S M I L$ encoded multimedia presentations [15] can require chiefly two types of resources:

- The audio channel that permits the delivery of the sound provided by an audio media.

- A region that determines a certain area of the screen wherein a media presentation has to be displayed. Thus, each non audio media (e.g. text, image or video) is associated with one among the defined regions. 
However, although these resources are important for processing a presentation, they are not mandatory, namely the multimedia presentation can occur even though one of the resource is unavailable, but without providing all the information vectors driven by the presentation. For instance, a video can be shown in its target area but its accompanying sound might not be delivered if the sound device is unavailable, and the converse is likely to happen also if the region is used by another media presentation. Hence, we call these kind of resources "non mandatory resources".

Besides, several media might be in conflict for the same resource (region, audio channel). In this case, basing on the functioning of the most famous $S M I L$ compatible player; Real one player [12] sets an ascending priority following the order of appearance of the media tags in the $S M I L$ document. Therefore, a media that uses a certain resource (audio channel or region) during its presentation might get it withdrawn if another conflicting media with higher priority starts its own in the meantime. In addition, Real one player is quite restrictive when processing a presentation, since a media could not recover the required resource if not acquired at its beginning, even though the missing resource is released before its ending.

However, theses dysfunctions could be controlled providing the applying of a formal methodology that makes it possible to detect and to correct them earlier during the authoring of the document, and to more efficiently manage them later at the playing stage. With this intention, we have proposed in a recent work a $T P N$-based model [3] that allows to capture these complex semantics. This model provides mechanisms that allows to specify accurately the behaviors induced by the allocation schemes of these kind of resources. For that effect, a non mandatory resource noted $r s$ is specified by the tuple $\left(f r(r s), b s_{i}(r s) i=1 . . p\right)$, where:

- $f r(r s)$ is a special place denoting when marked that $r s$ is free;

- $b s_{i}(r s) i=1 . . p$ is a set of places such that each place $b s_{i}(r s)$ is associated with a media $O_{i}$ that requires $r s$, and denotes once marked that $r s$ is held by the presentation $O_{i}$.

Remark: For a given marking, only one place among $\left\{f r(r s), b s_{1}(r s) . . b s_{p}(r s)\right\}$ should be marked.

In order to distinguish between a standard place and the one devoted to the modeling of a non mandatory resource (called "resource place"), we represent the latter with a double line circle. Moreover, these places behave differently comparing to standard places; their complex semantics are handled thanks to the use of the "preemptor hyperarc" [3]. The latter allows linking as an input a set of resource places to one transition. It uses two types of simple arcs: a strong arc represented as a continuous oriented arc, and a violator arc represented as a doted oriented arc. Hence, being given a preemptor hyperarc, the place linked through its violator arc is called a "violator resource place", whereas the place linked through its strong arc is called a "strong resource place" (See Fig .3).

To be effective, the "preemptor hyperarc" is supported with new event-concepts associated with the firing of a transition, given as follows:

- A strong event noted $t$, denoting the event generated when firing a transition $t$ such that the required resource is free.

- A violated event noted $t^{*}$ denoting the event generated when firing the transition $t$ such that its required resource is not free; held by a higher priority transition.

- A violator event noted ${ }^{*} t$ denoting the event generated when firing $t$ such that its required resource was violated from a lesser priority transition.

Therefore, the firing of the transition $t$ needs that $t$ be already enabled ${ }^{6}$, and its time constraints are consistent regarding the ones of other enabled transitions, in the same manner as in TPN [11]. Then, a strong event $t$ is generated when firing a transition $t$, if its strong resource place is marked. Otherwise, the violated event $t^{*}$ is generated, if both strong and violator resource places

\footnotetext{
${ }^{6}$ All its input standard places are marked.
} 
are unmarked. Finally, the violator event ${ }^{*} t$ is generated if the strong resource place is unmarked, and there is at least one violator resource place, which is marked.

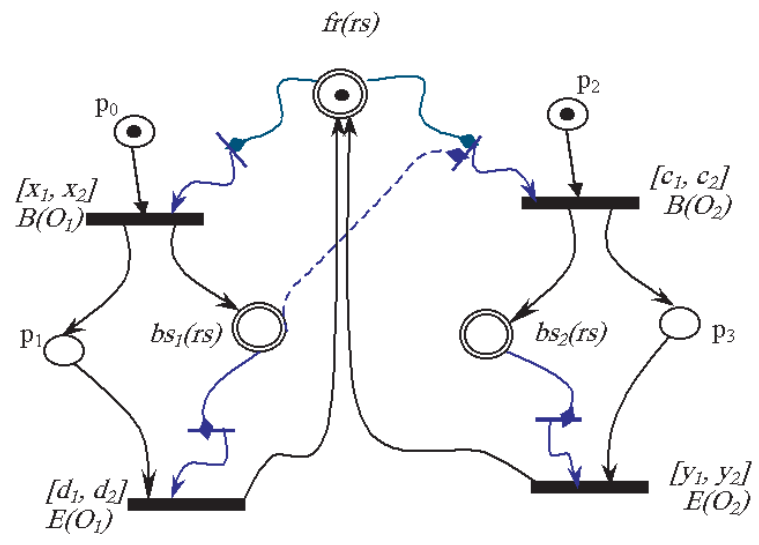

FIGURE 3: Real one player functioning based modeling

Remark:We assume that each preemptor hyperarc can contain at most one strong arc and each resource place contains at most one token. Moreover, we admit that an occurrence of a violated event $t^{*}$ can't produce a token in its output resource place.

To make clear the semantic of the previous mechanisms, we show thereafter how to model the requirements of two media presentations ${ }^{7} O_{1}$ and $O_{2}$ which are in conflict for a non mandatory resource $r s$.

Firstly, note recalling that according to Real one player-like functioning [12], if a media could not get the resource at the start, or had to give it up during its presentation, it would not be able to recover it afterwards, even though the resource is released before the end of its presentation. Considering that, the modeling of two presentations $O_{1}$ and $O_{2}$ in conflict for the resource $r s$ (according to Real one player- like functioning), is given by the specification depicted in Fig.3 that uses four preemptor hyperarcs:

- Two of them are connected to transitions $B\left(O_{1}\right)$ and $B\left(O_{2}\right)$ specifying that both media $O_{1}$ and $O_{2}$ need the availability of the resource $r s$ to start their presentations ${ }^{8}$. Otherwise, if the resource $r s$ is unavailable for one of the two presentations ${ }^{9}$ then only $\mathrm{O}_{2}$ which has priority (modeled using the violator arc linked to the resource place $b s_{1}(r s)$ ), might withdraw $r s$ from $O_{1}$, violating thus the resource $r s^{10}$.

- The other two preemptor hyperarcs are linked to the transitions $E\left(O_{1}\right)$ and $E\left(O_{2}\right)$ denoting that the presentations of $O_{1}$ and $O_{2}$ need to hold the resource $r s$ during the totality of the presentation. Hence, if the resource place $b s_{1}(r s)$ is unmarked when firing $E\left(O_{1}\right)$, a violated event is generated, denoting that the resource $r s$ has been withdrawn from $O_{1}$ by $O_{2}$ during its presentation. Notice that in the example of Fig 2, only $E\left(O_{1}\right)$ can be violated since $\mathrm{O}_{2}$ has priority. To model the case where no presentation has priority,(i.e., $\operatorname{Pr}\left(O_{1}\right)=\operatorname{Pr}\left(O_{2}\right)$ ) we need to add a violator arc going from the resource place $b s_{2}(r s)$ to the transition $B\left(O_{1}\right)$, namely both presentations can preempt each other

In addition, this modeling can be generalized for more than two conflicted medias. For instance, let $O_{1}, O_{2}, \ldots, O_{p}$ be a set of medias that are conflicting for the same resource $r s$, while assuming that $\operatorname{Pr}\left(O_{1}\right) \ldots \prec \operatorname{Pr}\left(O_{i}\right) \ldots \prec \operatorname{Pr}\left(O_{p}\right)$. To model the general case, we need to consider first the set of resource places $\left(f r(r s), b s_{i}(r s) i=1 . . p\right)$ where we assume that $f r(r s)$ is marked initially and connected as an input to all transitions $B\left(O_{i}\right)(i=1 . . p)$ by using strong $\operatorname{arcs}^{11}$. Then, each

\footnotetext{
${ }^{7}$ Where it is assumed that $O_{1}$ has less priority than $O_{2}$.

${ }^{8} \mathrm{~A}$ strong event is generated if the place $f r(r s)$ is marked

${ }^{9}$ The resource place $f r(r s)$ is unmarked and one of the two resource places $\left\{b s_{1}(r s), b s_{2}(r s)\right\}$ is marked

${ }^{10}$ The violator event ${ }^{*} B\left(O_{2}\right)$ is generated since the violator resource place $b s_{1}(r s)$ is marked

${ }^{11}$ It denotes that the media $O_{i}\{i=1 . . p\}$ need the availability of $r s$ at their start
} 


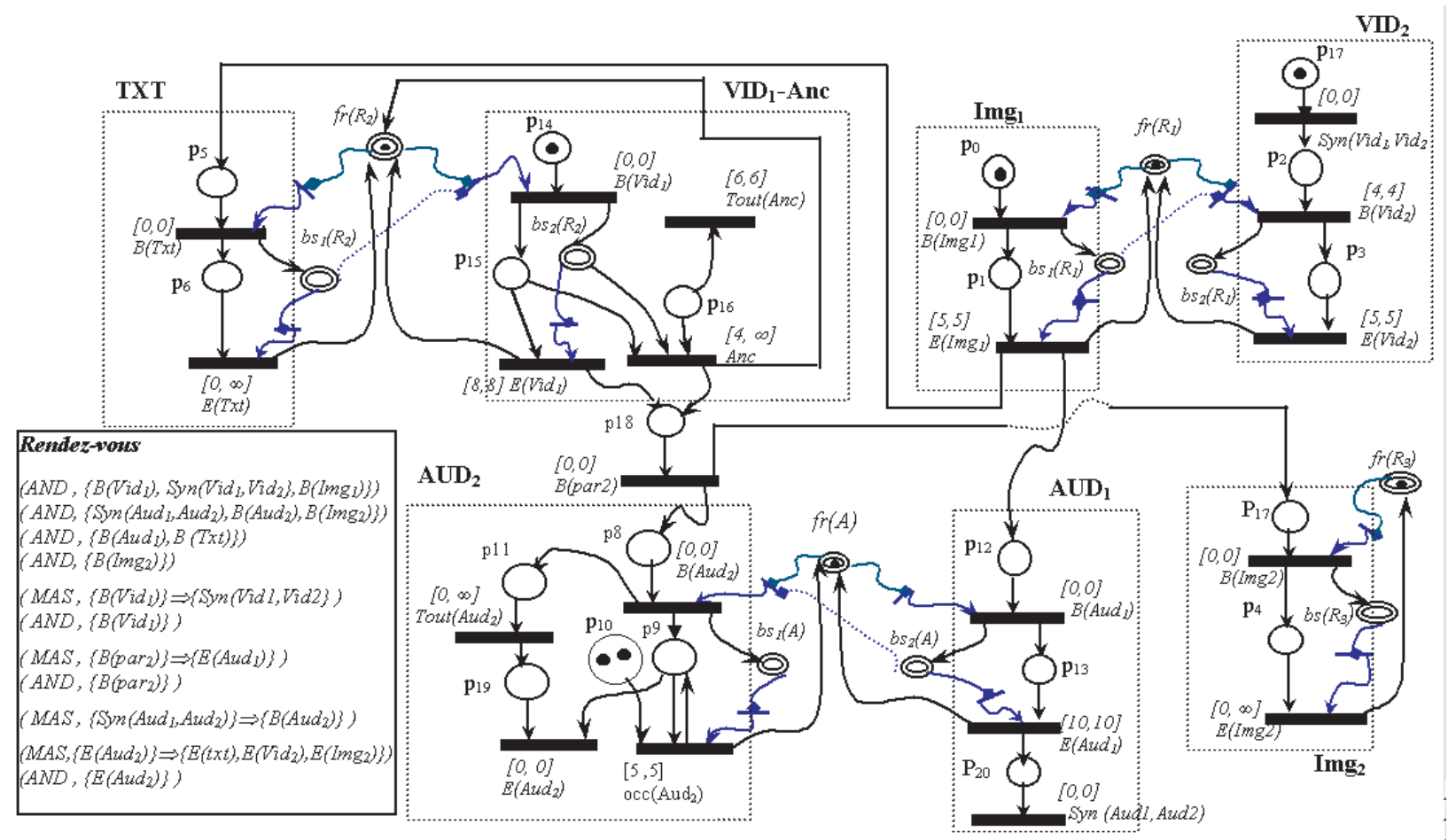

FIGURE 4: The STPTPN modeling the SMIL presentation requirements, according to a Real One Player-like functioning.

resource place $b s_{i}(r s)$ is connected as an output to transition $B\left(O_{i}\right)$ using standard arc, and as an input to $E\left(O_{i}\right)$ using the strong arc of the preemptor hyperarc. However, if $b s_{i}(r s)$ is marked; this denotes that the resource $r s$ is held by the media $O_{i}$. Besides, to model the preemption we need to link each transition $B\left(O_{j}\right)\{j=1$..p $\}$ to all resource places $b s_{i}(r s)$ (when $i \prec j$ ), by using the violator arcs of the preemptor hyperarc. The violator arcs give the right to $B\left(O_{j}\right)$ to violate the transitions of lesser priority when the resource $r s$ is held by one of them ${ }^{12}$. Otherwise, if the resource is held by a higher priority media $(i \succ j), B\left(O_{j}\right)$ can not obtain the resource since there is no violator arc linking the resource place $b s_{i}(r s)$ to the transition $B\left(O_{j}\right)$.

To make clear even more this modeling process, let us consider again our work example. The time and the synchronization requirements as well as the resource allocation schemes can be modeled by the STPTPN specification [3] depicted in Fig.4. The modeling of the time requirements of a basic media $O$ is based on the use of two key transition events, its starting event noted $B(O)$ and its ending event noted $E(O)$. In addition, these events can be temporally constrained according to the time requirements linked to the media. Then, each media is described as a TPN unit, and the different units are connected according to the structuring of the SMIL document. We add thereto rendezvous that allows to express the simultaneous firing of a set of transitions according to the Master or to the And synchronization schemes. Finally, the resource allocation schemes are described by resource places and by using preemptor hyperarc when it is needed. By the way, we can determine from the code of the SMIL document that three regions $R_{1}, R_{2}$ and $R_{3}$ are defined. The region $R_{1}$ is devoted to $I m g_{1}$ and $V i d_{2}$ whereas the region $R_{2}$ is dedicated to medias $V i d_{1}$ and Txt. Finally, the region $R_{3}$ is used by the media $I m g_{2}$. Hence, basing on a Real one player like functioning the media $\mathrm{Vid}_{1}, \mathrm{Aud}_{2}$, and $V i d_{2}$ have priority on respectively, Txt, $A u d_{1}$, and $I m g_{1}$.

From this specification, we are able to derive the reachability graph when using the enumeration technique developed in [5]. We obtain the graph depicted in Fig.5.a wherein temporally inconsistent scenarios have been left out. As a matter of fact, we seek for analyzing how behave

\footnotetext{
${ }^{12}$ When there is one among the resources places $\left\{b s_{i}(r s)\{i=1 . . j-1\}\right.$ which is marked.
} 
the different scenarios (already proven as temporally consistent), when considering besides the resource conflict issue. Therefore, we can notice that media $\mathrm{Vid}_{2}$ is forced to violate the region $R_{1}$ from $I m g_{1}$ to initiate its own presentation; this occurs at date $4 \mathrm{~s}$, and it is denoted by the occurrence of the violator event ${ }^{*} B\left(V i d_{2}\right)$ labeling the edge $(1-7)$. This configuration entails (whatever the considered temporally consistent scenario), that $I m g_{1}$ will not be shown for the last second of its presentation which is highlighted by the generation of the violated event $E\left(I m g_{1}\right)^{*}$ stamping the edges $(8-13)$ and $(7-12)$.

In other respects, worth noting that media $V i d_{1}$ that acquires initially the region $R_{2}$ is in position to keep it until its ending, preventing thus the media $T x t$ to be visualized despite that $V i d_{1}$ will restore the region before that $T x t$ will end. These cases are illustrated by the scenarios denoted by the paths $(0 \longrightarrow 26 \longrightarrow 20)$ and $(0 \longrightarrow 22 \longrightarrow 20)$. Therefore, $T x t$ is not able to acquire the region during its whole presentation which is denoted by the occurrence of the violated events $B(T x t)^{*}$ respectively $E(T x t)^{*}$ labeling the edges $(12-21)$ respectively $(25-20,30-20)$. On the other hand, only the scenario given by the path $(0 \longrightarrow 13 \longrightarrow 20)$ makes it possible to show $T x t$, if the user interaction intervenes at date $5 s$; achieving $V i d_{1}$ before that $T x t$ starts.

\section{QUALITATIVE CONSISTENCY OF MULTIMEDIA PRESENTATION}

We propose thereafter to revisit the consistency concept introduced in [13] by considering besides the resource conflict issue. This issue will be worked out when assuming a Real one playerlike functioning thanks to the modeling process introduced in [4]; hence, a new methodology verification is thus introduced. In order to stand the latter we need first to give some useful notations.

Notation 4.1 Let $\mathrm{Gr}$ be the reachability graph obtained from the STPTPN specification [3] and $S$ be a path in $\operatorname{Gr}$ starting from the initial node. We note $\operatorname{Seq}(O, S)$ given as the event sequence $e_{1} \stackrel{s}{\rightarrow} e_{2}$, the run observed in $S$ starting from the event denoting the beginning of $O$ (given by $e_{1}$ ), to the one denoting its ending (given by $e_{2}$ ). We have $\operatorname{Seq}(O, S)=\varnothing$ if $O$ does not occur in $S$.

We endeavor now to analyze all potential behaviors that could be induced from the modeling process already developed for that purpose [4], and briefly introduced in the previous Section. With this intention, we give next all possible runs of a basic media in terms of event sequences $\operatorname{Seq}(O, S)$ while providing each run with its interpretations according to a Real one player-like functioning.

- $B(O) \stackrel{s}{\rightarrow} E(O)$ : denoting a consistent execution of $O$ with resource acquisition during its whole presentation.

- $B(O) \stackrel{s}{\rightarrow} E(O)^{*}$ : denoting the case where the resource was acquired at the start of $O$ before that it has been withdrawn by a higher priority media without having the possibility to recover it afterwards.

- $B(O)^{*} \stackrel{s}{\rightarrow} E(O)^{*}$ : specifying the situation where $O$ is not able to acquire the resource during the whole presentation.

- ${ }^{*} B(O) \stackrel{s}{\rightarrow} E(O)$ : denoting the case where $O$ has violated the missing resource from a lesser priority media to start its own presentation, while being able to keep it till its ending.

- ${ }^{*} B(O) \stackrel{s}{\rightarrow} E(O)^{*}$ : Specifying the case where $O$ has to violate the required resource to start before it was forced to give it up definitely to a higher priority presentation.

According to the previous interpretations, one can remark that consistent runs of a media are those that induce the event sequences $B(O) \stackrel{s}{\rightarrow} E(O)$ or ${ }^{*} B(O) \stackrel{s}{\rightarrow} E(O)$. Therefore, "a multimedia presentation is without resource conflicts, if its corresponding graph does not contain any occurrence of a violated event". In other words, a multimedia presentation can be appreciated as qualitative if there is no media processed therein that has been prevented from being perceived for a part or for the totality of its execution.

However, one can notice that we cannot adopt the same policy for continuous media (e.g. Video, animation, or audio) as for discrete media (e.g. Text or image). To be explicit, a discrete media determines a still image or a text that needs to be shown only for a part of its presentation to let 


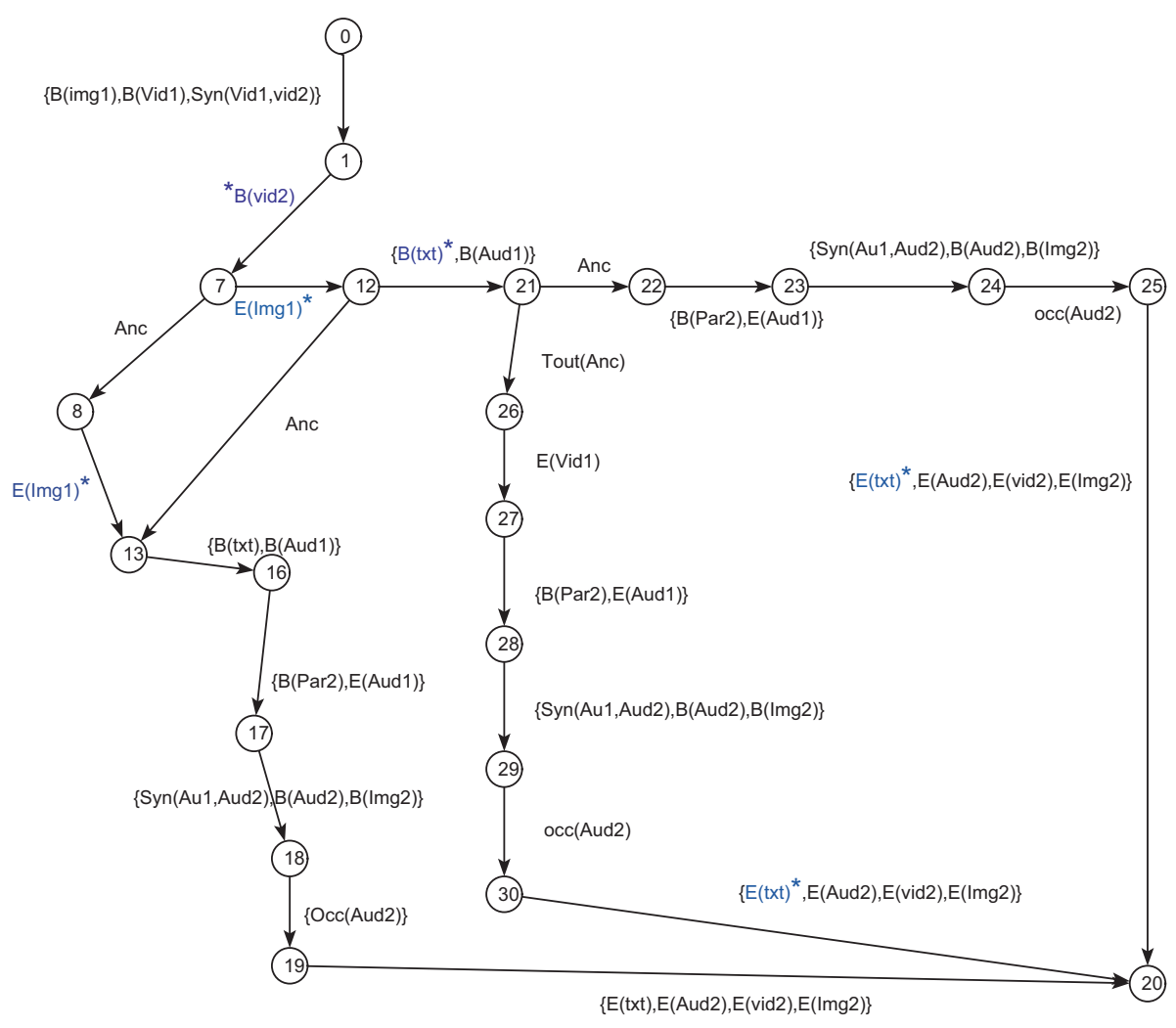

(a)

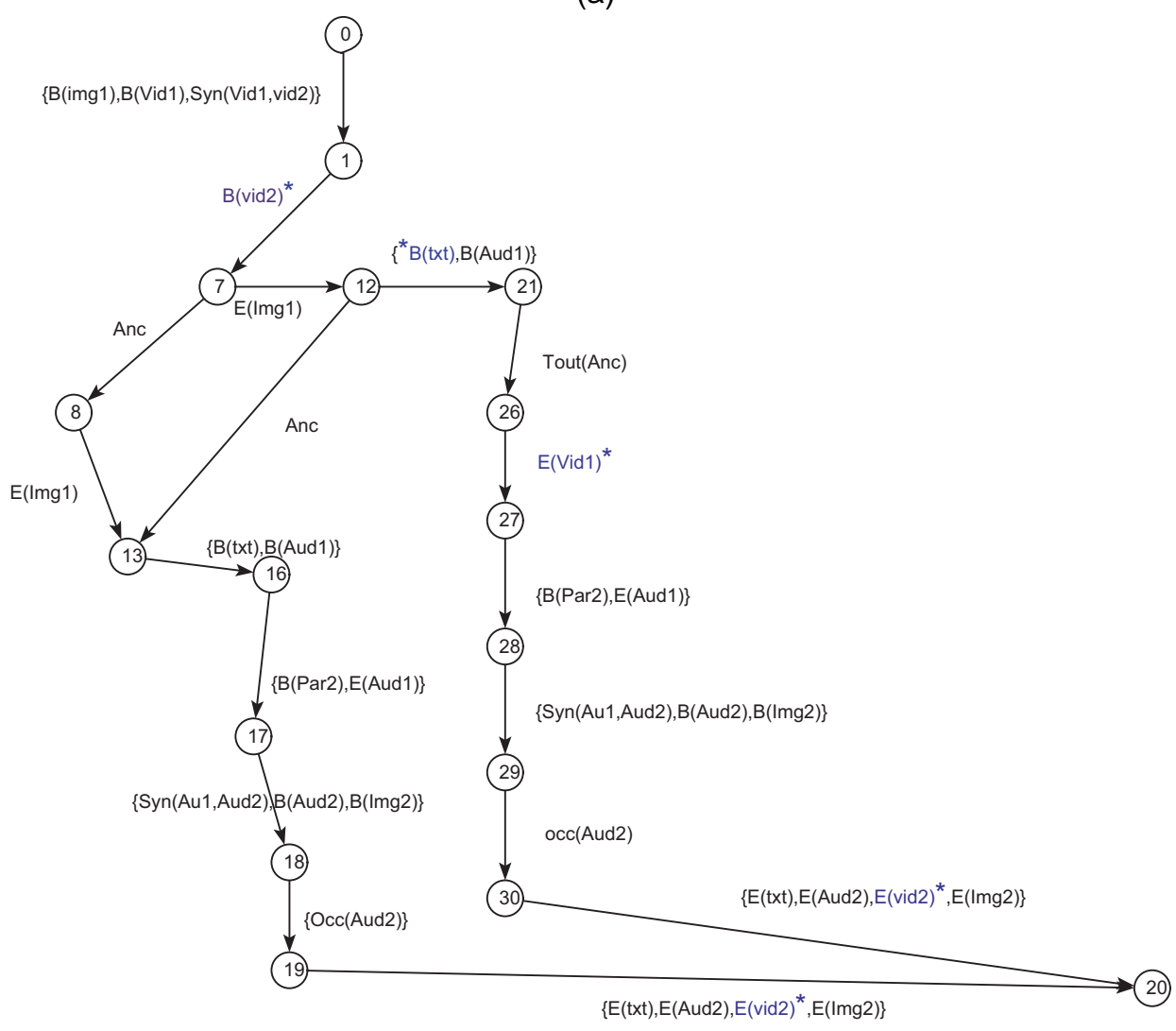

(b)

FIGURE 5: The reachability graphes depicting in addition the resource constraints of the SMIL multimedia presentation. 
the driven information be perceived by the user, whereas missing a part of a continuous media may entail a loss of information that could be hardly restored to the user.

Considering that, we define thereafter a new consistency paradigm called "qualitative consistency" that aims to revisit the previous concept by extending it to the resource conflict verification.

Definition 4.1 (Qualitative consistency). A multimedia presentation is said to be "qualitatively consistent" according to Real one player-like functioning, if its corresponding graph $\mathrm{Gr}$ is "temporally consistent" and for every path $S$ in $G r$ and for each media $O$ processed in $S$, we have:

- If $O$ is a discrete media (i.e. image or text) then $\operatorname{Seq}(O, S) \neq B(O)^{*} \stackrel{s}{\rightarrow} E(O)^{*}$.

- If $O$ is a continuous media (i.e., animation, video, or audio ) then $\operatorname{Seq}(O, S) \in$ $\left\{B(O) \stackrel{s}{\rightarrow} E(O),{ }^{*} B(O) \stackrel{s}{\rightarrow} E(O)\right\}$.

The later definition states that a "qualitatively consistent" presentation permits for discrete medias to be shown only for a part of their durations while it needs that continuous medias must be delivered for the totality of their durations. To be explicit, as regards discrete media we need to prove that all discrete media can at least be user-perceived for a part of their presentations. In the context of Real one player-like functioning, we need then to check that the event sequences linked to each discrete media are not equal to $B(O)^{*} \stackrel{s}{\rightarrow} E(O)^{*}$. As regards continuous medias, we should verify that continuous medias have to be user-perceived for their whole presentations. Therefore, basing on the interpretations already given in the context of a Real one player-like functioning, a media presentation is perceived for the totality of its duration if its event sequence is equal to $B(O) \stackrel{s}{\rightarrow} E(O)$ or to $* B(O) \stackrel{s}{\rightarrow} E(O)$.

So, the qualitative consistency verification is performed by exploiting the reachability graph. Therefore, if the graph does not conform to the Definition 4.1, we could cure the document by identifying the origin of the inconsistencies while locating the media involved in the resource conflict. Hence, we can remove the situations of conflicts either by adding new regions or by temporally formatting the document to avoid their appearance. This methodology could be used at the authoring stage in building a consistent multimedia document by exploiting the reachability graph derived from its formal specification. On the other hand, at the player stage the tool could proceed to schedule consistent scenarios when they exists. In case where all the paths are inconsistent, the player should warn the user of resource conflict existence.

In other respects, the reachability graph can be also used in the computing of a more precise access pattern that could be efficiently exploited in managing a pre-fetching scheme or an adapted delivery mechanism in the same manner as we have proposed for standard approaches [1]. With this intention, the request scheduler has to point out the discrete medias that will not be perceived because of resource conflicts and to avoid thus to request them. Also, the scheduler should request only the portions of continuous medias that are able to be perceived; the graph allows therefore to determine which media is concerned by this specific treatment and the portions to be requested.

To make clear our proposal, let us consider again our work example. So, when applying the newly defined concept on the derived graph thereof given in Fig.5.a, we notice that the multimedia presentation offers one possible "qualitatively consistent scenario" depicted by the path $(0 \longrightarrow$ $13 \longrightarrow 20)$. In this case, only the discrete media $\operatorname{Img}_{1}$ is still hidden for its last ending second while it is shown for the four first seconds. However, the other two scenarios remain qualitatively inconsistent since the media $T x t$ is prevented to be displayed for the totality of its duration. Hence, the whole multimedia presentation is qualitatively inconsistent too.

To illustrate even more the impact of resource conflict on the consistency verification. Let us consider now the same presentation but while assuming the reverse priority order, namely the medias $T x t, A u d_{1}$, and $I m g_{1}$ have priority on respectively $V i d_{1}, A u d_{2}$, and $V i d_{2}$. Hence, the 
reachability graph corresponding to the formal specification is given in Fig.5.b. When comparing the latter with the one of Fig.5.a, one can notice that the path $22 \longrightarrow 20$ is no more existing ${ }^{13}$. In concrete terms, the media $T x t$ which has priority may preempt $V i d_{1}$, if the latter does not end before date $5 \mathrm{~s}$. Thus, $V i d_{1}$ will be prevented to be displayed (between $5 s$ and $8 s$ ) and therefore the user can no longer click on the associated link. So, the occurrence of ${ }^{*} B(T x t)$ stamping the edge $(12-21)$ denotes the preemption of $R_{2}$ from $V_{i d}$ and this happens only in the scenario depicted by the path $0 \longrightarrow 21 \longrightarrow 20$. On the other hand, the scenario given by the path $0 \longrightarrow 13 \longrightarrow 20$ describes the case where the user interacts at date $5 s$ just in time to end $V i d_{1}$ before the start of Txt.

Furthermore, whatever the considered scenario, the media $V i d_{2}$ is prevented to be shown for the totality of its presentation since the region $R_{1}$ is detained by $I m g_{1}$ when $V i d_{2}$ starts. Note recalling that in accordance with Real one player functioning, the media $V i d_{2}$ can not recover the region ${ }^{14}$ even after its release at date $5 \mathrm{~s}$.

Remark: We model the need of the region for the occurrence of the link in Fig. 4 by connecting the resource places $B s_{2}\left(R_{2}\right)$ in the input and $f r\left(R_{2}\right)$ in the output of the transition Anc; this denotes that the user interaction needs the availability of the resource region to occur. One has to notice that traditional approaches that deal only with temporal consistency (as it is shown in the graph of the Fig.2) do not take into account the impact of the unavailability of a region for the occurrence of an interaction. It results thereof that the modeling process admits that the user interaction may occur even though the associated region could not be provided for the anchor period. Hence, these methodologies may induce additional scenarios that could skew the consistency verification.

\section{CONCLUSION}

The paper proposed to revisit the concept of multimedia document consistency by considering the resource issue in the verification process. This methodology is based on deriving the reachability graph from a TPN-based specification already introduced in [3]. This framework makes it possible thanks to the refining of event concepts (strong event, violated event, violator event), to capture the resource allocation schemes of complex multimedia presentations.

Hence, with the support of the modeling process developed in [4] while assuming a Real one player-like functioning, we have achieved a thorough analysis of all possible runs in terms of event sequences. The latter has permitted us to rethink and to extend the consistency verification methodology so that it becomes possible to detect the incoherences owed to resource conflicts when they occur.

This newly defined methodology allows within the authoring and player tools to highlight the inconsistencies induced by resource-conflict situations. Besides, the graph can be used to extract a more efficient and precise access-pattern that could be exploited in implementing an adapted pre-fetching scheme.

Further work will lead us to extend the qualitative consistency paradigm to other player functioning and while including other concerns dealing with the quality of the presentation.

\section{REFERENCES}

[1] A.Abdelli and N.badache. A Semantic based pre-fetch scheme for SMIL presentation Proxydelivery 12th IEEE MMM-Multimedia modelling conference, Beigin China 4-6 Jan. 2006 Page(s):8 pp.

[2] A.Abdelli and M.Daoudi "Towards a SMIL document analysis using an algebraic time net," 5th PCM 2004, Tokyo, Japan, Nov-30- LNCS - Vol 3333/2004.

[3] A.Abdelli and N.Badache: Synchronized Transitions Preemptive Time Petri Nets: A new model towards specifying multimedia requirements; In proc of IEEE ACS/AICCSA, Dubai

\footnotetext{
${ }^{13}$ This path denotes the scenario wherein the user interaction may intervene between $5 \mathrm{~s}$ and $6 \mathrm{~s}$

${ }^{14}$ Since $V i d_{2}$ was not able to acquire the resource at its beginning.
} 
March 8, 2006 Page(s):17-24

[4] A.Abdelli and N.badache. Toward Specifying Multimedia Requirements Using a New Time Petri Net Model. International Journal CIT, 2007 (to appear).

[5] A.Abdelli and N.badache. Une Approche pour la Construction de l'Espace d'Etat d'un Réseau de Petri à Flux. In proc of 7Th MCSEAI International Maghrebian Conference on software engineering and Artificial intelligence. Agadir Morocco 7-9 December 2006.

[6] Ambulant Player http://ambulantplayer.org.

[7] J.P.Courtiat, M.Diaz. R.C De Oliveira P.Senac. Proving temporal consistency in a new multimedia synchronisation model. In proc of ACM Multimedia- Boston Nov 96.

[8] GRiNS http://www.oratrix.com/grins/

[9] Chung-Ming Huang, Chian Wang, and Chih-Hao Lin,"Interactive Multimedia Synchronization in the Distributed Environment Using the Formal Approach," IEE Proceedings - Software, VOL. 147, NO. 4, pp. 131-143, August 2000.(NSC 89-2213-E-006-049) (EI)

[10] N.Layaïda, L.Sabry-Ismail, and C.Roisin, "Dealing with Uncertain Durations in Synchronized Multimedia Presentations" MTAP Kluwer-Vol.18,No.3, pp213-231, 2002.

[11] P. Merlin. A study of the recoverability of computer system. PHD Thesis Dep. Computer. Science, Univ. California, Irvine, 1974.

[12] Real Player Home page Url: http://real.com.

[13] P.N.M.Sampaio, C.A Sontos, J. P. Courtiat. Using formal method to verify the temporal semantics of SMIL documents. In Proc. Conference of Software: Theory and Practice, IFIP World Congress, Beijing, Chine, August $21-252000$.

[14] C.A.S.Santos, J.P. Courtiat, P. Saqui-sannes. A disign methodology for the formal specification and verification of hypermedia documents. In Proc of FORTE/ PSTV'98 Paris France, Nov1998. Chapman\&Hall.

[15] W3C Recommendations, SMIL2.0 Specification. URL:http://www.w3.org/TR/SMIL20 